\title{
A rare case as different cause of retropharyngeal and spinal epidural abscess: spondylodiscitis
}

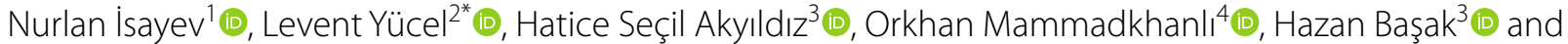 \\ İhsan Doğan ${ }^{5}$
}

\begin{abstract}
Background: Retropharyngeal abscess (RPA) is a life-threatening, dangerous condition and uncommon in adults. The coexistence of RPA, cervical spinal epidural abscess (CSEA), and spondylodiscitis is extremely rare.

Case presentation: We present a case with a retropharyngeal and epidural abscess caused by spondylodiscitis. A 61-year-old man was referred to our clinic with the complaints of sore throat, limitation in neck range of motion, numbness, and weakness in the left arm and the left ear for one month. The airway was not obstructed. Neurological deficits were detected in his left arm. Cervical computed tomography revealed a $50 \times 30 \times 15 \mathrm{~mm}$ retropharyngeal abscess. Cervical magnetic resonance imaging showed abscess, C5-6 spondylodiscitis and epidural abscess, and myelopathic signal changes in the C3-7 spinal cord. The abscess was drained, and C5-6 discectomy was performed. The patient was discharged with cervical collar and antibiotics.
\end{abstract}

Conclusions: Multidisciplinary approach that consists of otolaryngologist, neurosurgeon, and infectious disease specialist is needed to avoid complications and any delay.

Keywords: Discitis, Epidural abscess, Retropharyngeal abscess

\section{Background}

Retropharyngeal abscess (RPA) is a life-threatening, dangerous condition and uncommon in adults. The incidence of RPA in adults is unclear; however, it is 4.1 per 100,000 in pediatric population [1]. Trauma in adults and suppurative lymphadenitis after upper respiratory tract infections in children (especially under 5 age) are the main causes of RPA. Diabetes, poor oral hygiene, intravenous drug abuse, and immunodeficiency are the other reasons for RPA [2, 3]. Cervical spine epidural abscess (CSEA) is also a rare condition with the incidence of 19.6 per 100,000 [1]. The risk factors are similar with RPA. Head and neck infections were historically the main cause of
CSEA; however, neurosurgical procedures are, nowadays, a common reason [4].

Spondylodiscitis encompasses osteomyelitis of the vertebrae and intervertebral discs. Different names for this condition have been used to describe such as vertebral osteomyelitis, spinal osteomyelitis [5]. The incidence of spondylodiscitis is 7.4 per 100,000 , and coexistence of RPA, CSEA, and spondylodiscitis is extremely rare [1]. Besides life-threatening conditions in these patients, permanent neurological defects can occur if spondylodiscitis is not treated.

We present a case with a retropharyngeal and epidural abscess caused by spondylodiscitis.

\footnotetext{
*Correspondence: leventyucel51@hotmail.com

${ }^{2}$ Department of Otorhinolaryngology, University of Health Sciences,

Gülhane Training and Research Hospital, Ankara, Turkey

Full list of author information is available at the end of the article
} 


\section{Case presentation}

A 61-year-old man was referred to our clinic with complaints of sore throat, limitation in neck range of motion, numbness, and weakness in the left arm and the left ear for 1 month. There was no fever or dysphagia. Hyperemia in the oropharynx, tenderness, and pain in the left lateral of the neck with deep palpation were detected in his physical examination. Furthermore, detailed neurological examination showed hypoesthesia in the left arm, and the muscle strength of the left arm was 4 of 5 at flexion and 2 of 5 at extension and the muscle strength of left fingers was 2 of 5 at abduction and adduction. His previous medical history was free from diabetes mellitus, trauma, or any other medical diseases. The fiberoptic examination disclosed that the airway was not obstructed; however, the posterior wall of the pharynx was swollen and hyperemic.

The peripheral white blood cell count was $6860 / \mathrm{mm}^{3}$, C-reactive protein was elevated to $32 \mathrm{mg} / \mathrm{dl}$, and sedimentation was $90 \mathrm{~mm} / \mathrm{h}$. A cervical contrast-enhanced computed tomography $(\mathrm{CT})$ revealed a $50 \times 30 \times 15 \mathrm{~mm}$ abscess at the retropharyngeal area anterior to the C3-7 vertebrae, heterogeneity at the corpus of the $\mathrm{C} 5-7$ vertebrae, a slight loss of height at the C6 vertebra, and a lytic formation with a diameter of $1 \mathrm{~cm}$ in the $\mathrm{C} 7$ vertebra (Fig. 1). Cervical vertebrae magnetic resonance imaging (MRI) showed RPA, C5-6 spondylodiscitis and epidural abscess, and myelopathic signal changes in the C3-7 spinal cord (Fig. 2). Besides, lytic formation in the $\mathrm{C} 7$ vertebra was thought of as a hemangioma.

The empirical antibiotic therapy (cefepime, $3 \mathrm{~g}$, three times a day and teicoplanin, $400 \mathrm{mg}$, once a day) was started immediately. The patient was transferred to a neurosurgery clinic for surgery for both abscess and

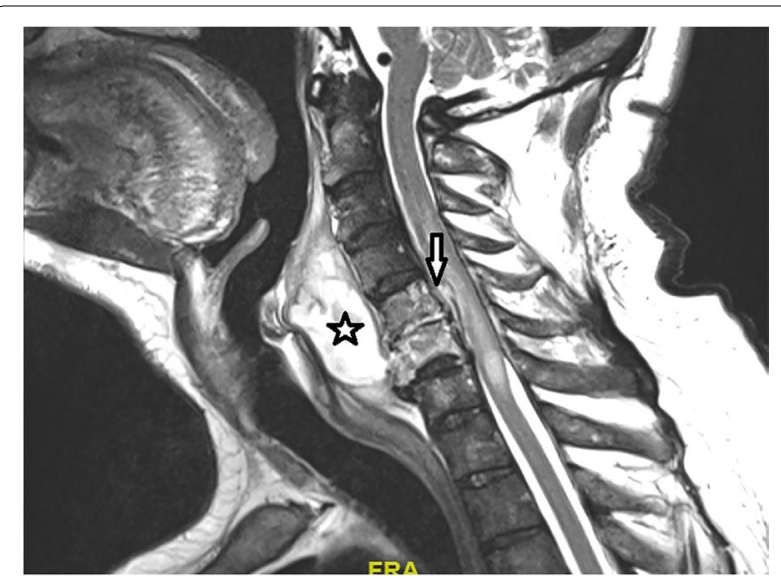

Fig. 2 T2-weighted, sagittal section of vertebra MRI: the star shows the retropharyngeal abscess, and the arrow show the spinal epidural abscess. Myelopathic signal changes of the spinal cord are clearly seen

spondylodiscitis. Intraoperatively, the abscess was drained and sent for culture, tuberculosis polymerase chain reaction (PCR), and Brucella agglutination. C5-6 discectomy was also performed with an anterior cervical approach. There was no complication at the intraoperative or postoperative period. Neck stabilization with a cervical collar was recommended.

The pus culture, Brucella agglutination, and tuberculosis PCR results were negative. Methicillin-resistant Staphylococcus epidermidis reproduced in blood culture. However, it was decided that it was contamination from the skin. Histopathological examination revealed only inflammation. The patient discharged one week after the operation with ciprofloxacin $(750 \mathrm{mg}$, twice a day) and

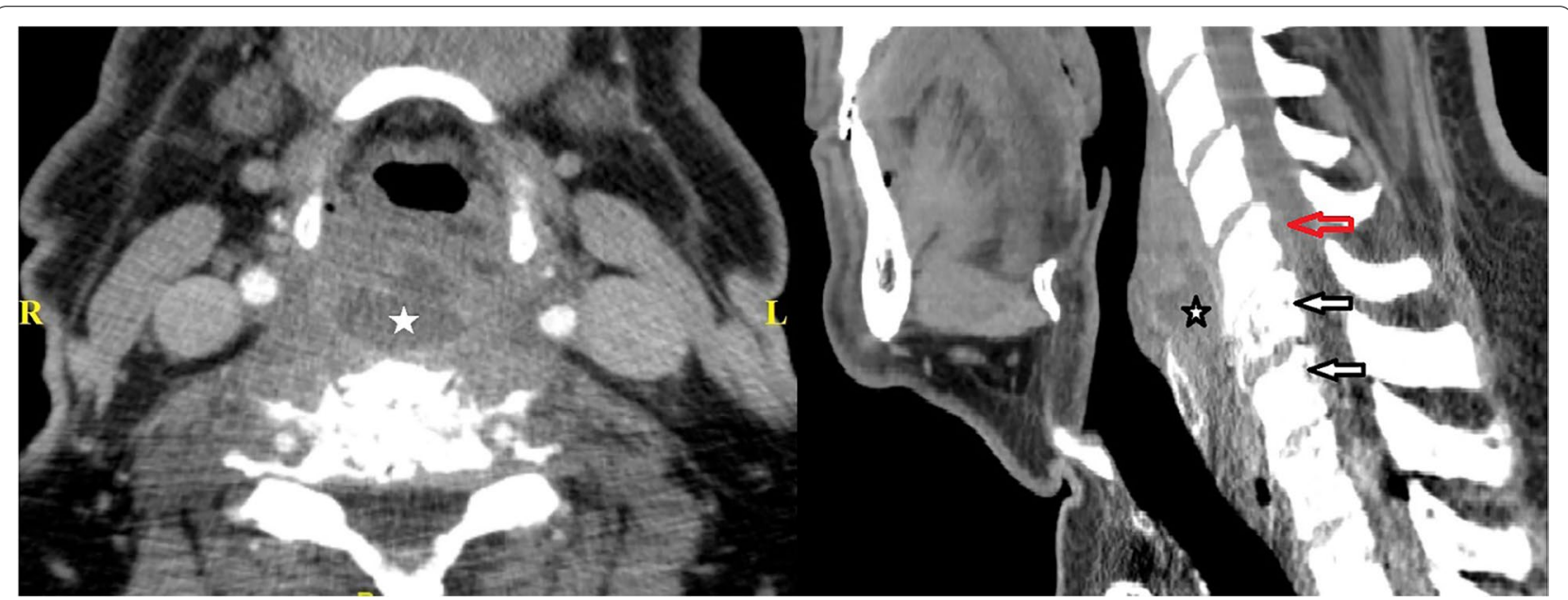

Fig. 1 Axial and sagittal sections of CT: the stars show the retropharyngeal abscess, and the arrows show the degeneration of C5-6 vertebrae 
teicoplanin (400 mg, once a day) according to suggestions from a specialist of infectious diseases. The total antibiotic use after discharge was 42 days. When the medical therapy ended, the muscle strength of the left arm and left fingers were 4 of 5 ; however, hypoesthesia was still going on. Physical therapy was recommended to the patient. Control cervical MRI was performed postoperative second year to the patient and showed myelopathic signal changes at the level of the C5-6 vertebra (Fig. 3). The patient had no complaint at postoperative 4.5 years, the muscle strength of the left arm and fingers were $4-5$ of 5 , and he had minimally hypoesthesia on his left arm.

\section{Discussion}

The three layers of cervical fascia enclose the contents of the head and neck and occur in spaces for potential infections. The fascia of the neck limits the direct spread of infection; however, the connections between spaces cause spread of infection to this defined anatomical areas. Therefore, this is the basis of mortal complications of deep neck infection. The retropharyngeal space extends from the skull base down to the mediastinum, and it is bounded by carotid sheaths laterally, buccopharyngeal fascia anteriorly, and prevertebral fascia posteriorly $[1,6]$.

Trauma from foreign bodies or surgical instruments is the major cause of RPA, and nontraumatic RPA is very rare in adults. This is because of the regression of

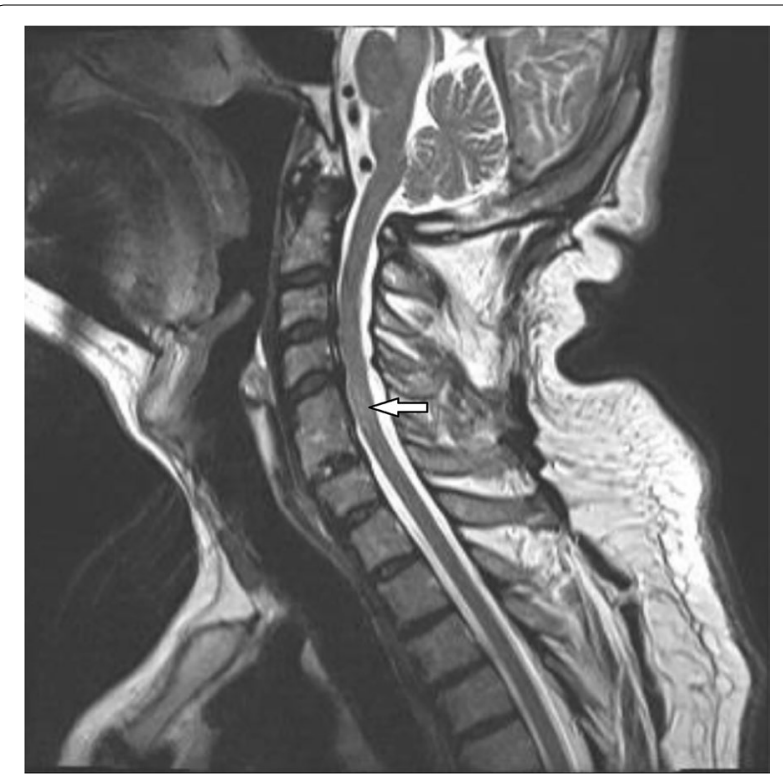

Fig. 3 Postoperative second year control, T2-weighted, sagittal section of vertebra MRI: the arrow shows the myelopathic signal changes of the spinal cord retropharyngeal lymph nodes at the age of 4-5 years. Therefore, RPA is more frequent before 4-5 years. Nontraumatic RPA is mainly caused by immunodeficiency such as diabetes, human deficiency virus, intravenous drug abuse, and cirrhosis [7].

Cervical spondylodiscitis also known as cervical vertebral osteomyelitis $(\mathrm{CVO})$ is a rare and serious condition that causes permanent neurological deficits. It is expected that abscess formation like CSEA and RPA is a complication of $\mathrm{CVO}$ because of the proximity of these structures. The cervical region is less common than the thoracic or lumber region; however, the mortality and morbidity are more frequent than others [5].

The RPA is often caused by polymicrobial agents, and Streptococcus pyogenes, Staphylococcus aureus, and Haemophilus influenzae are more common agents that are responsible for RPA [8]. The empiric use of antibiotic therapy before surgical drainage may be the reason not to reproduce any agent in our case.

The diagnosis of RPA with CSEA and CVO starts with a suspicion of paravertebral infection. Sore throat, dysphagia, "hot patato" voice, restriction and pain on neck movements, fever, dyspnea, stridor, tachypnea, and neurological deficits are the most common complaints of RPA with CVO and CSEA [1, 6]. Neurological impairment of CVO is between 68 and 80\% [7]. Therefore, the patient is questioned for neurological deficit before RPA symptoms. Cervical CT is the most useful radiological examination for the diagnosis of RPA; however, it is necessary to perform MRI to make certain diagnosis of RPA, CSEA, and CVO and to exhibit the compression to the spinal cord without any delay to avoid mortal and morbid complications such as mediastinitis, carotid rupture, sepsis, jugular venous thrombosis, and permanent neurological deficits [6].

The treatment strategy for RPA, CVO, and CSEA consists of both medical therapy and surgery. Furthermore, tuberculosis must be excluded. Drainage of abscess, debridement and decompression of the spinal canal, and cervical stabilization are the often used surgical approach [9]. The anterior approach is the preferable choice for these coexistences [1]. The use of long-term antibiotics according to culture results is also needed.

\section{Conclusions}

$\mathrm{CVO}$ is an extremely rare cause of coexistence with RPA and CSEA; however, the mortal and morbid complications make them very important. Multidisciplinary approach that consists of otolaryngologist, neurosurgeon, and infectious disease specialist is needed to avoid complications and any delay. 


\section{Abbreviations}

RPA: Retropharyngeal abscess; CSEA: Cervical spine epidural abscess; CT: Computed tomography; PCR: Polymerase chain reaction; MRI: Magnetic resonance imaging; CVO: Cervical vertebral osteomyelitis.

\section{Acknowledgements}

None.

\section{Authors' contributions}

Conception-L.Y., N.I. H.S.A., and O.M. Design-N.I., H.S.A., and O.M. Supervision-H.B. and I.D. Fundings - not applicable. Data collection and/ or processing - L.Y.,N.I., H.S.A., and O.M. Analysis and/or interpretation-L.Y. and N.I. Literature review-L.Y., N.I., H.S.A., O.M., H.B., and I.D. Writing of the manuscript-L.Y. and N.I. Critical review-L.Y., N.I., H.S.A., O.M., H.B., and I.D. All authors have read and approved the final version of the manuscript.

\section{Funding}

This study did not receive any specific grant from funding agencies in the public, commercial, or not-for-profit sectors.

\section{Availability of data and materials}

Not applicable

\section{Declarations}

Ethics approval and consent to participate

Not applicable.

\section{Consent for publication}

Written informed consent form the participant was obtained for publication of this case report and accompanying images.

\section{Competing interests}

The authors declare that they have no competing interests.

\section{Author details}

${ }^{1}$ Department of Otorhinolaryngology, HB Guven Clinic, Baku, Azerbaijan.

${ }^{2}$ Department of Otorhinolaryngology, University of Health Sciences, Gülhane Training and Research Hospital, Ankara, Turkey. ${ }^{3}$ Department of Otorhinolaryngology, Ankara University Faculty of Medicine, Ankara, Turkey. ${ }^{4}$ Department of Neurosurgery, Yuksek Ihtisas University School of Medicine, Medical Park Ankara Hospital, Ankara, Turkey. ${ }^{5}$ Department of Neurosurgery, Ankara University Faculty of Medicine, Ankara, Turkey.

Received: 11 May 2021 Accepted: 11 October 2021

Published online: 16 November 2021

\section{References}

1. Epstein N (2020) Diagnosis, and treatment of cervical epidural abscess and/or cervical vertebral osteomyelitis with or without retropharyngeal abscess; a review. Surg Neurol Int. 11:160

2. Jain A, Singh I, Meher R, Raj A, Rajpurohit P, Prasad P (2018) Deep neck space abscesses in children below 5 years of age and their complications. Int J Pediatr Otorhinolaryngol. 109:40-43

3. Joshua J, Scholten E, Schaerer D, Mafee MF, Alexander TH, Crotty Alexander LE (2018) Otolaryngology in critical care. Ann Am Thorac Soc. 15(6):643-654

4. Eggart MD, Greene C, Fannin ES, Roberts OA (2016) A 14-year review of socioeconomics and sociodemographics relating to intracerebral abscess, subdural empyema, and epidural abscess in southeastern Louisiana. Neurosurgery. 79(2):265-269

5. Almansour H, Pepke W, Akbar M (2020) Pyogenic spondylodiscitis. Orthopade. 49:482-93

6. Vieira F, Allen SM, Stocks RMS, Thompson JW (2008) Deep neck infection. Otolaryngol Clin North Am. 41(3):459-483

7. Sakaguchi A, Ishimaru N, Ohnishi H et al (2017) Retropharyngeal abscess with cervical discitis and vertebral osteomyelitis caused by Escherichia coli in a patient with liver cirrhosis. Infez Med 25(2):169-173

8. Tollard E, Choussy O, Bertrand M, Thiébot J, Dacher JN, Marie JP (2007) Prevertebral abscess mimicking a retropharyngeal abscess and revealing a double-location spondylodiscitis: case report. J Neuroradiol. 34(2):141-143

9. Giordan E, Marton E, Scotton G, Canova G (2019) Outcomes and risk factors for spontaneous spondylodiscitis: case series and meta-analysis of the literature. J Clin Neurosci. 68:179-187

\section{Publisher's Note}

Springer Nature remains neutral with regard to jurisdictional claims in published maps and institutional affiliations.

\section{Submit your manuscript to a SpringerOpen ${ }^{\circ}$ journal and benefit from:}

- Convenient online submission

- Rigorous peer review

- Open access: articles freely available online

- High visibility within the field

- Retaining the copyright to your article

Submit your next manuscript at $\boldsymbol{\nabla}$ springeropen.com 